VIII ${ }^{\text {èmes }}$ Journées Nationales Génie Civil - Génie Côtier, Compiègne, 7-9 septembre 2004

\title{
Compréhension du phénomène hydraulique développé par une digue partielle et reconstitution des efforts globaux à partir de la mesure des efforts locaux.
}

\author{
Christophe Colmard(a), René Bouchet(b), Isabelle Lesprit(c)
}

\author{
(a)Ingénieur principal: Naval Architecture \& Maritime Civil Works Saipem S \\ 1/7 Avenue de San-Fernando 78884 Saint Quentin Yvelines Cedex France. Tel: 0161378428 \\ (b)Conseiller technique auprès du département des travaux publics de la Principauté de Monaco \\ Service des Travaux Publics 8, rue Louis NOTARI MC 98000 MONACO \\ Tel : 37793158000 fax : 37793158604 \\ (c)Ingénieur expérimentation Océanide \\ Océanide Port de Brégaillon 83502 La Seyne-sur mer Tel 0494300117
}

Résumé.

La société SAIPEM-SA (anciennement Bouygues Offshore) concessionnaire exclusive du "Mur d'Eau Fixe" déposé par la Principauté de Monaco [1], a conduit diverses études, pour en améliorer le fonctionnement [2]. Dans cet article nous montrons comment il nous a été possible de comprendre les phénomènes hydrauliques mis en jeux par le design qui a été retenu pour la construction de la contre-jetée installée à Monaco en 2002. A partir du dépouillement des essais réalisés par Océanide, il a été possible d'établir la contribution de chaque effort local à l'évolution des efforts globaux. Le détail des différentes phases importantes caractérisant le phénomène hydraulique est montré pour la houle centennale.

\begin{abstract}
SAIPEM-SA (formerly Bouygues Offshore) is the exclusive concessionaire of the " Fixed Water Wall » patent [1]. It is a Monaco’s patent. SAIPEM-SA develops a new amelioration based on the "Fixed Water Wall" system [2]. This profile amelioration has been used in the design of the counter jetty (installed in Monaco in 2002). The model tests had been performed by Oceanide (2000). In this paper, the wave loads study of the partial breakwater is explained and we show that it is possible to compare the global wave load with the superposition of the local loads. The detail of the hydraulic organization load is shown. The wave conditions are the 100 years return period.
\end{abstract}

Mots-clés: Efforts globaux, Efforts locaux, Mur d’eau Fixe, Bybop, Digue partielle

\section{Introduction.}

Les mesures ont été réalisées au 1/40em au laboratoire Océanide [3] dans le cadre du projet de la contre jetée de Monaco. La digue est soumise aux houles monégasques de secteur $160^{\circ}-75^{\circ}$ (par rapport au nord). La houle centennale est définie par $\mathrm{Tp}=9.6 \mathrm{~s}$ et $\mathrm{Hs}=4 \mathrm{~m}$. Le cas de houle régulière présenté dans cet article est $\mathrm{T}=10 \mathrm{~s}, \mathrm{Hi}=6.5 \mathrm{~m}$ et $\mathrm{h}=40 \mathrm{~m}$. Cette houle est représentative de la houle centennale monégasque. 


\section{Digue étudiée.}

La digue fait 150 mètres de long, 30 mètres de large et est immergée de 9 mètres. La profondeur d'eau est principalement de 40 mètres. Le profil de la digue est composé :

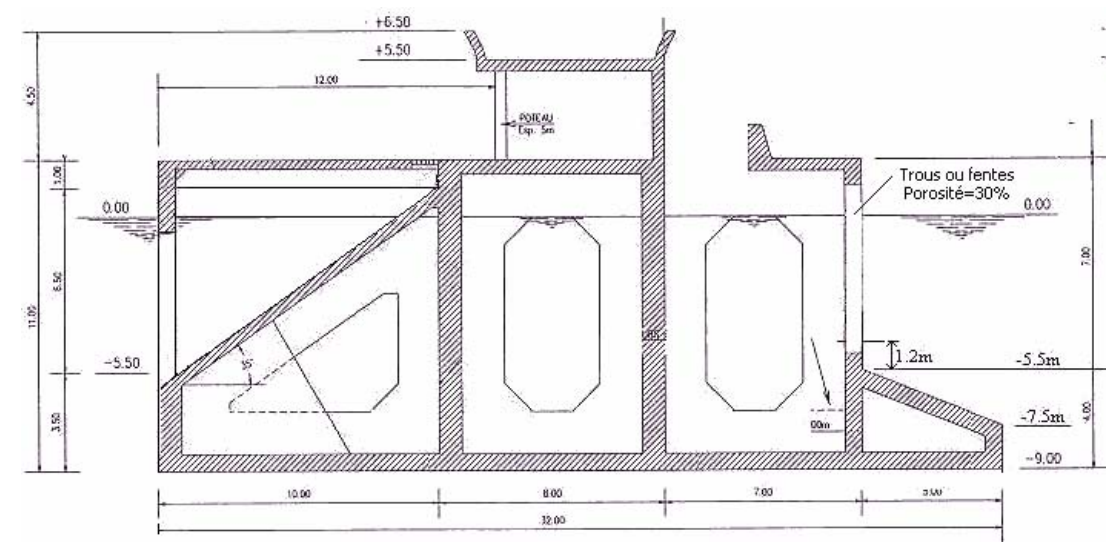

Figure. 1 Plan de la digue.

- d'un béquet avant nécessaire pour contrer les vitesses verticales en amont de la structure.

- d'une chambre d'atténuation munie d'une paroi perforée d'une porosité égale à 30 pour-cent.

- d'une chambre centrale

- d'un béquet arrière incliné à 35º nécessaire pour limiter le couplage masse d'eau du port masse d'eau du large (brevet Bybop [1]).

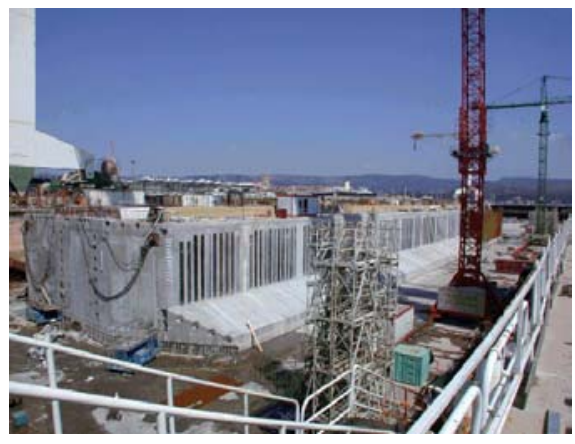

\section{Instrumentation.}

Les mesures de niveaux d'eau sont effectuées au moyen de douze sondes résistives dont deux sont installées en périphérie de la digue et deux dans la maquette (figures 2). La mesure des efforts globaux est réalisée au moyen d'une balance dynamométrique six composantes fixée sur la partie supérieure du caisson. Les fréquences propres d'oscillations de la maquette obtenues en modèle sont : $f(F x)=13.3 \mathrm{~Hz}, f(F z)=55.6 \mathrm{~Hz}$ et $f(M z)=20 \mathrm{~Hz}$. 

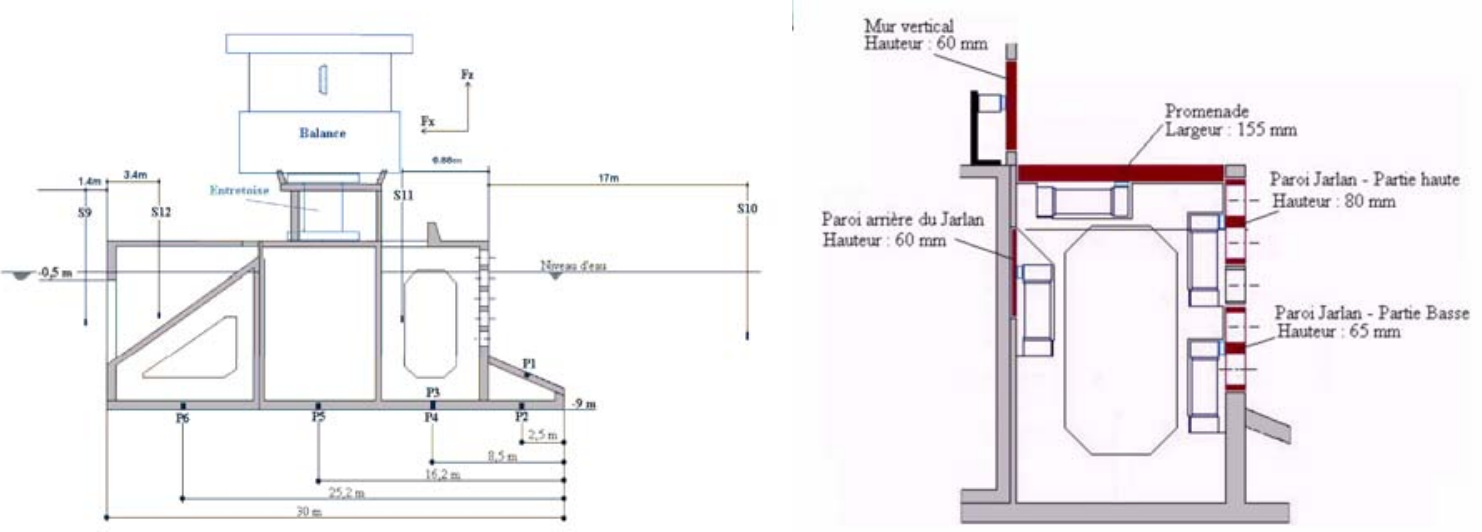

Figure 2 : Instrumentation de la digue

La mesure des efforts locaux est réalisée avec des capteurs d'efforts fixés sur des tronçons de paroi du caisson. Ces efforts sont mesurés par déformation de jauges de contrainte montées sur des barreaux parallélépipédiques rectangles qui travaillent en traction et compression.

Des mesures de pression sont réalisées en 6 points : sur le becquet amont (P1), sous le becquet amont (P2), dans la chambre Jarlan (P3), sous la chambre Jarlan (P4), sous le caisson central (P5), sous la partie aval de l'ouvrage (P6).

\section{Cycle de la houle sur l'ouvrage.}

Au début de l'ascension de la vague sur l'ouvrage, la masse d'eau vient frapper le béquet amont. La vague vient ensuite attaquer la paroi perforée avec une claque d'effort située sur la partie supérieure de ce mur. La vague se propage alors sur les superstructures de l'ouvrage et dans la chambre d'atténuation. Le mur arrière de la chambre est alors fortement sollicité (surtout dans sa partie haute).

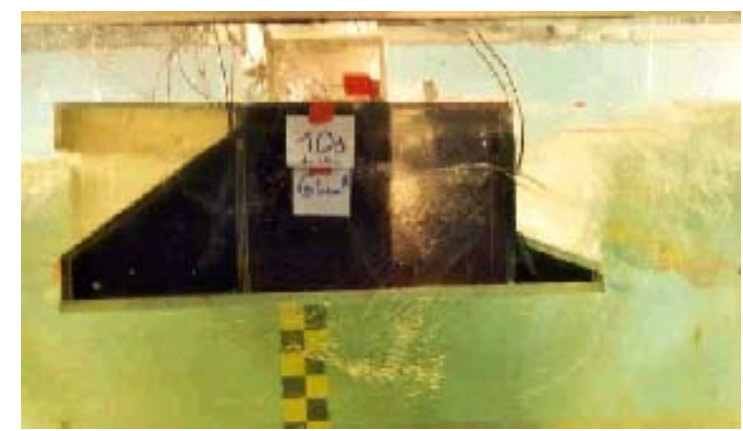

Figure. 3 L'impact de la vague sur le béquet amont

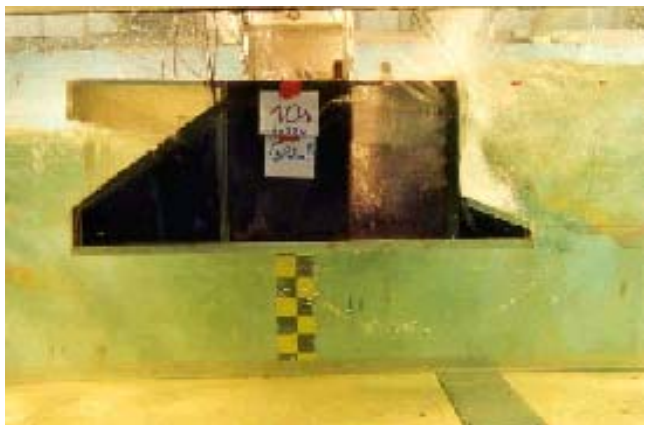

Figure. 4 L'impact sur la paroi perforée

La vague se retire ensuite en refluant à la fois sur les superstructures et dans la chambre d'atténuation. La chambre finit de se vidanger et une nouvelle vague est déjà en phase d'ascension. Les efforts dynamiques d'impact sont principalement horizontaux. La composante verticale est souvent de type hydrostatique. 


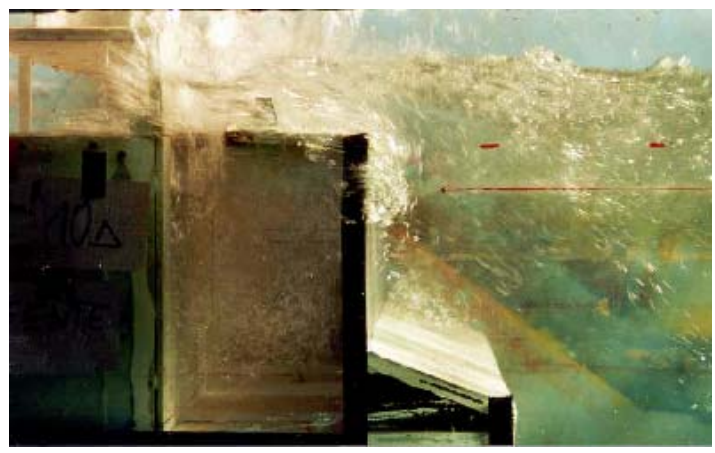

Figure. 5 L'impact sur le mur supérieur

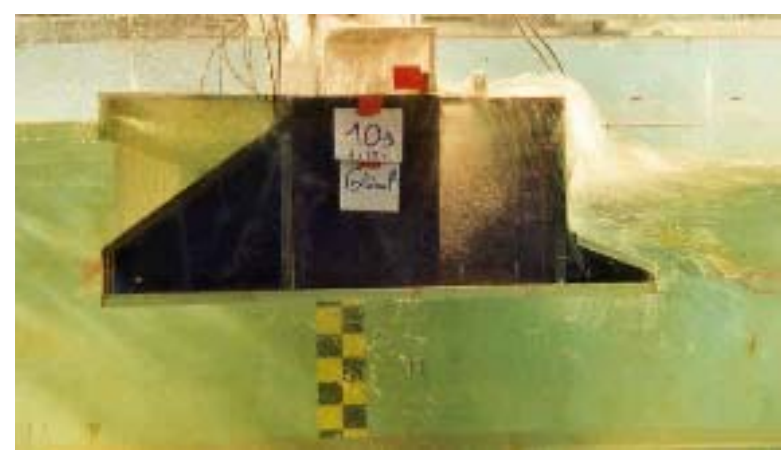

Figure.6 Reflux de la vague

\section{Etude des efforts.}

Nous avons découpé les surfaces de la digue partielle en plusieurs surfaces simples sur les quelles nous appliquons les efforts mesurés ponctuellement :
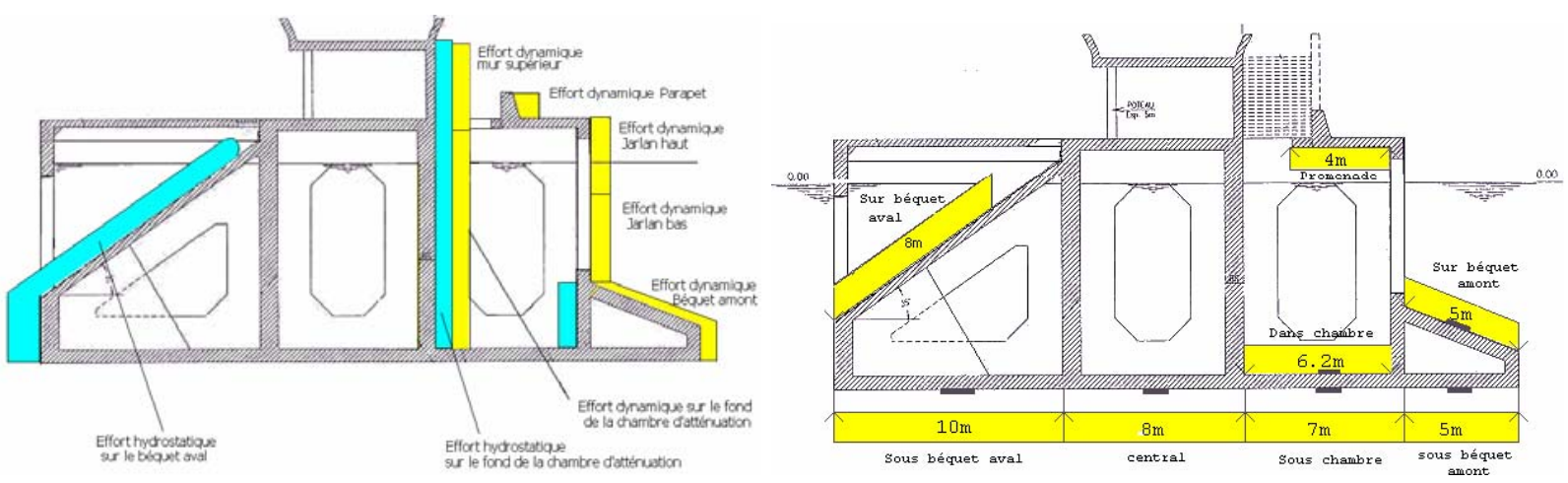

Figure. 7 Surfaces d'application des efforts horizontaux et des efforts verticaux

Pour cet essai, avec $9 \mathrm{~m}$ de tirant d'eau, $40 \mathrm{~m}$ de profondeur, $\mathrm{Hi}=6.5 \mathrm{~m}$ et $\mathrm{T}=10 \mathrm{~s}$ nous trouvons: Un $\mathrm{Kr}=0.25$ (coefficient de réflexion), $\mathrm{Kt}=0.09$ (transmission) $\mathrm{Fx} \max =750 \mathrm{~K}$ Newtons $/ \mathrm{ml}$ et $\mathrm{Fz} \max =500 \mathrm{~K}$ Newtons $/ \mathrm{ml}$.

\subsection{Traitement des inclusions d'air}

Le béquet amont est la première partie de la digue à être attaquée par la houle. C'est l'exemple que nous allons utiliser pour montrer la méthode que nous avons utilisée lorsqu'il se posait des problèmes d'inclusion d'air. L'estimation de cet effort en horizontal est réalisée grâce au capteur de pression P1. On constate sur la figure 8 que la mesure de pression est bruitée. Deux phénomènes importants sont à prendre en considération pour transférer la pression de l'échelle maquette à l'échelle réelle:

- Le phénomène d'inclusion d'air qui induit des vibrations à fréquence élevée $(>40 \mathrm{~Hz})$

- L'oscillation de la maquette $(13 \mathrm{~Hz})$, celle ci étant maintenu par ses superstructures. 

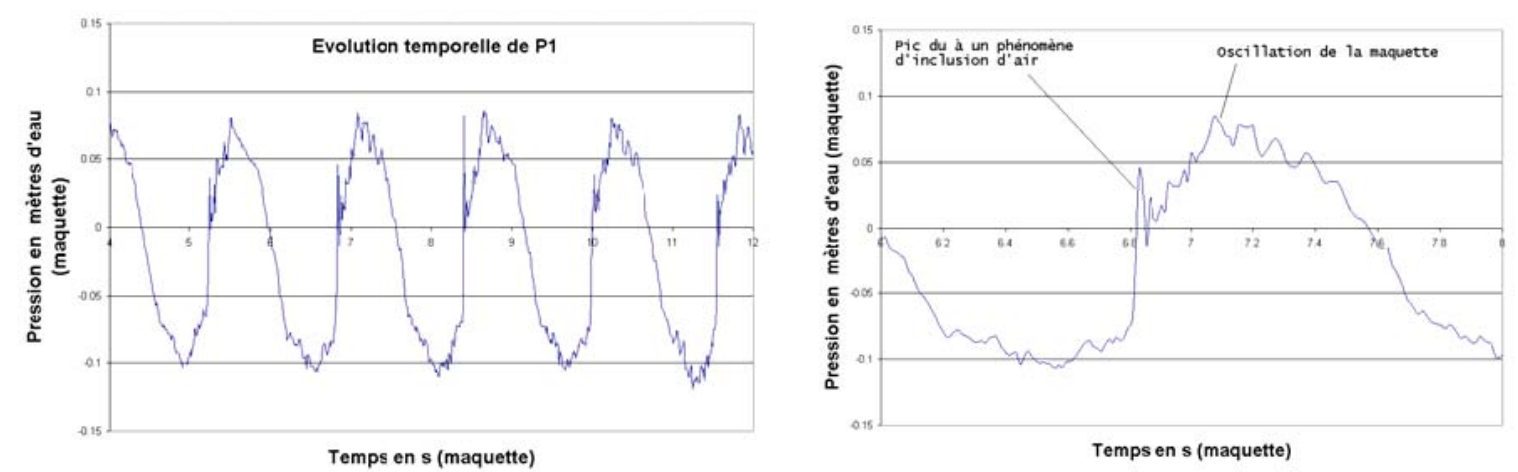

Figure. 8 Traitement de la pression P1.

On constate en observant les visualisations que les inclusions d'air proviennent initialement du reflux de l'eau de la chambre perforée (voir figure 9).

Lors de la phase de retrait une quantité importante d'air est piégée au dessus du béquet et les poches d'air occasionnent des pics de pression ponctuels de type "Compression shock" décrit par Lungreen [4].

Lungreen propose un modèle dit "Loi de compression" basé sur les travaux de Bagnold. La loi de similitude est bâtie à partir de l'équation suivante :

$\mathrm{Pmax}^{2 / 7}-1.4+0.4 \times \mathrm{Pmax}^{-5 / 7}=\mathrm{H}^{\prime \prime}$

Avec $\mathrm{H}^{*}$ la hauteur de vague addimentionnée.

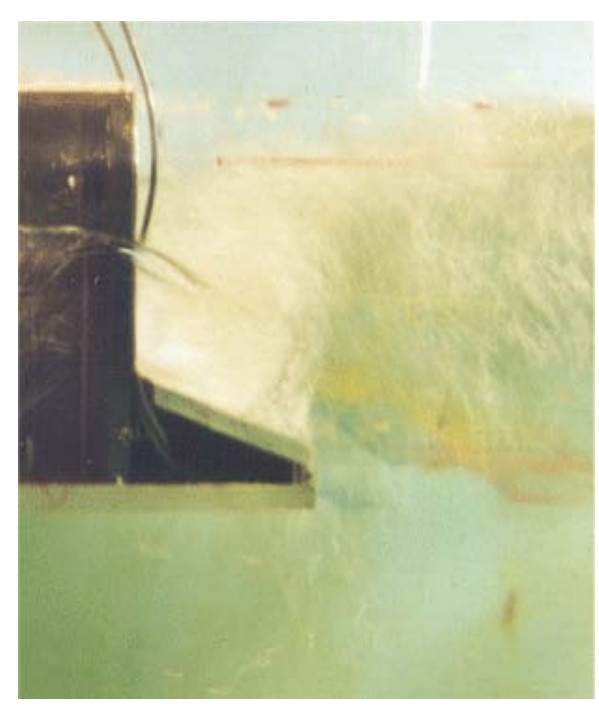

Figure. 9 Inclusion d'air. 
Pour passer à l'échelle réelle nous obtenons l'abaque de la figure 10:

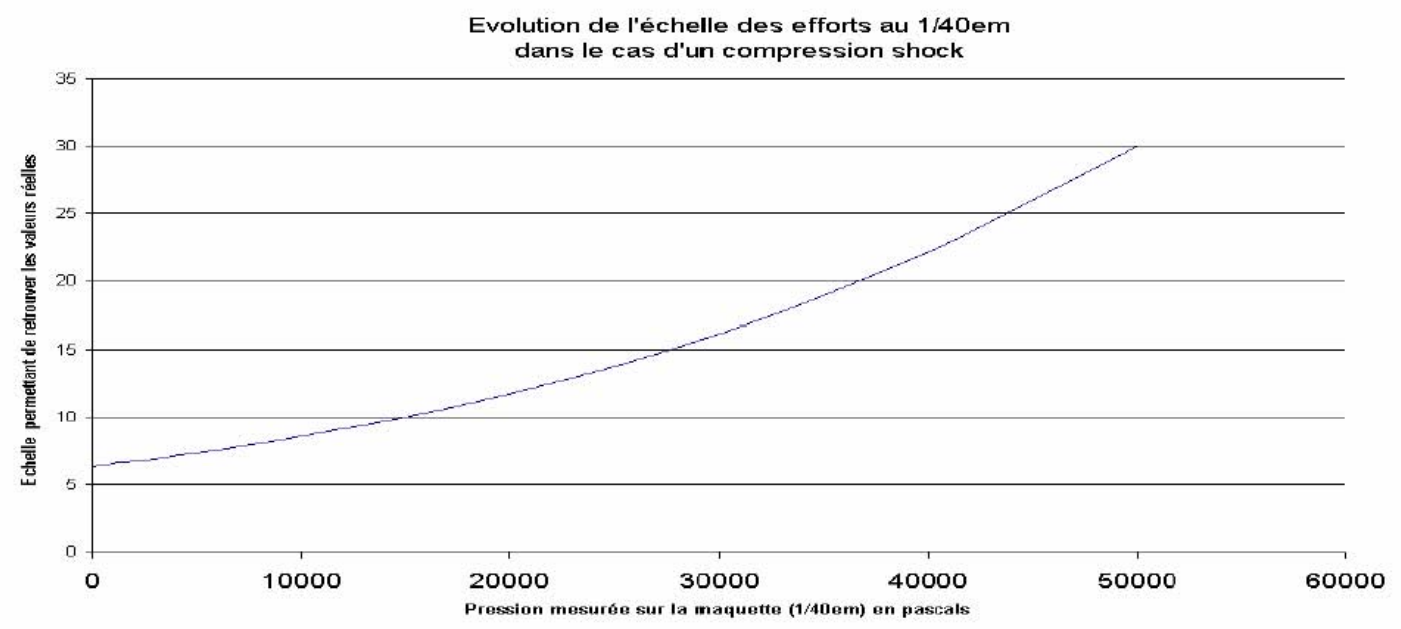

Figure. 10 Compression shock, échelle de Lungreen.

Cette échelle n'est utilisable que sur les pics générés par le phénomène d'inclusion d'air et non ceux dus à l'oscillation de la maquette. En filtrant le signal brut avec un filtre basse-bas à $20 \mathrm{~Hz}$ nous n'hottons au signal de pression que la partie liée au inclusion d'air. En hottant ce signal à la pression brut nous obtenons comme le montre la figure 11 la partie induite par les phénomènes d'inclusion d'air.

Nous obtenons la pression s'exerçant sur le béquet amont à l'échelle réelle en additionnant la partie sans inclusion d'air (filtrée à $10 \mathrm{~Hz}$ pour enlever l'influence de la balance) multipliée par l'échelle de Froude et le signal due aux inclusions d'air multiplié par l'échelle de Lungreen. On constate que les phénomènes de pic d'inclusion d'air deviennent très faibles lorsqu'ils sont passés à l’échelle réelle.
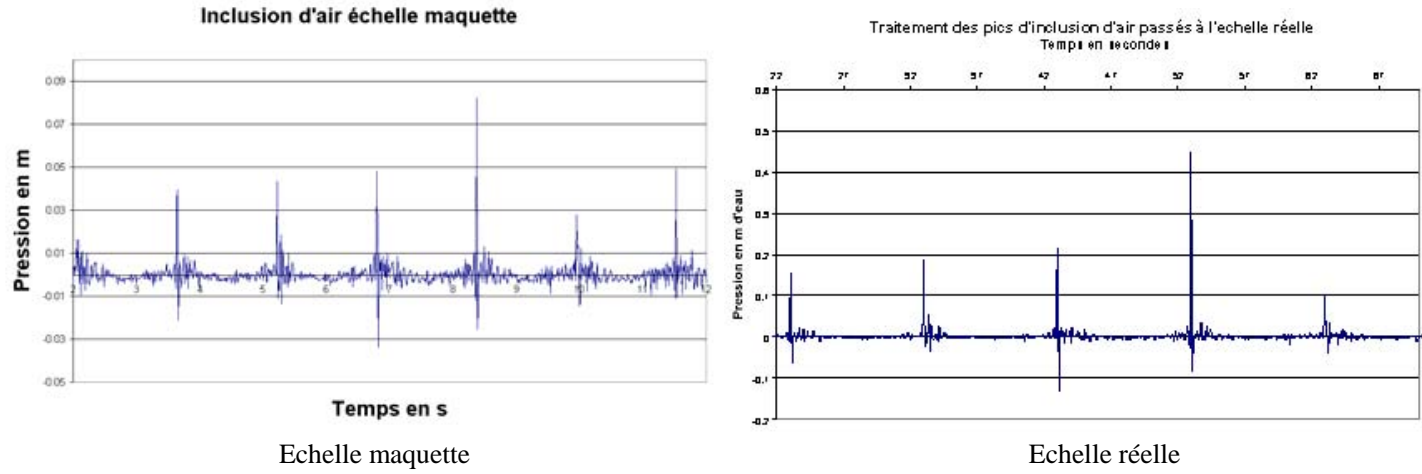

Figure 11 Pression due aux inclusions d'air.

\subsection{Effort sur le mur perforé}

Le second pic d'effort est localisé sur le mur Jarlan lorsque la vague incidente attaque l'ouvrage. Elle frappe d'abord le bas de la paroi puis la partie supérieure. La photo (figure 12) montre la violence de l'impact sur la partie supérieure. 
Les problèmes d'inclusions d'air sur les mur perforés sont beaucoup moins critiques pour deux raisons : La mesure n'est pas ponctuelle mais réalisée sur une large portion du mur d'où une moyenne des effets ponctuelles dus aux bulles et deuxièmement le mur est perforé ce qui minimise les effets d'inclusion.
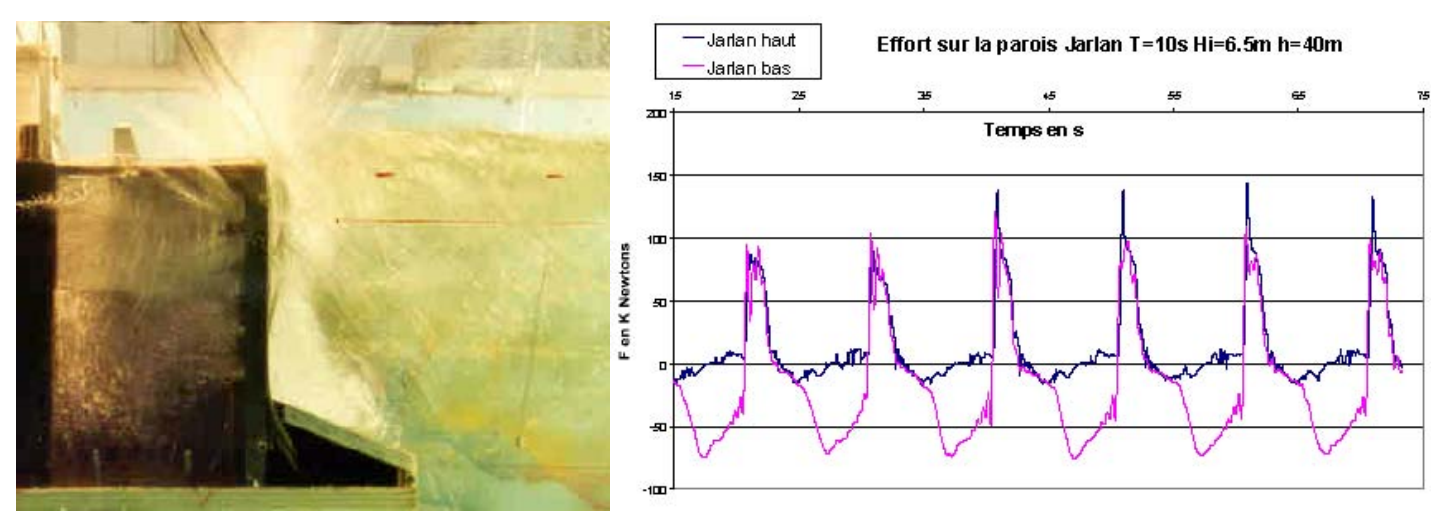

Figure 12 Impact sur le mur perforé.

\subsection{Effort sur mur du fond de la chambre Jarlan}

Le troisième choc est localisé sur le mur du fond de la chambre Jarlan. La vague est passée au travers et au dessus du mur perforé et frappe le mur suivant, 0.2 secondes plus tard (soit $1.25 \mathrm{~s}$ en réel), c'est à dire à peu près à la célérité de la houle. Le choc est concentré principalement sur la partie supérieur du mur (mur entre $+3.2 \mathrm{~m}$ et $+6.5 \mathrm{~m}$ ). Ce choc induit des phénomènes d'inclusion d'air qu'il faut prendre en compte. Nous réalisons le même traitement que pour le béquet amont. A cet effort il faut ajouter l'effort induit sur le mur par la hauteur d'eau.
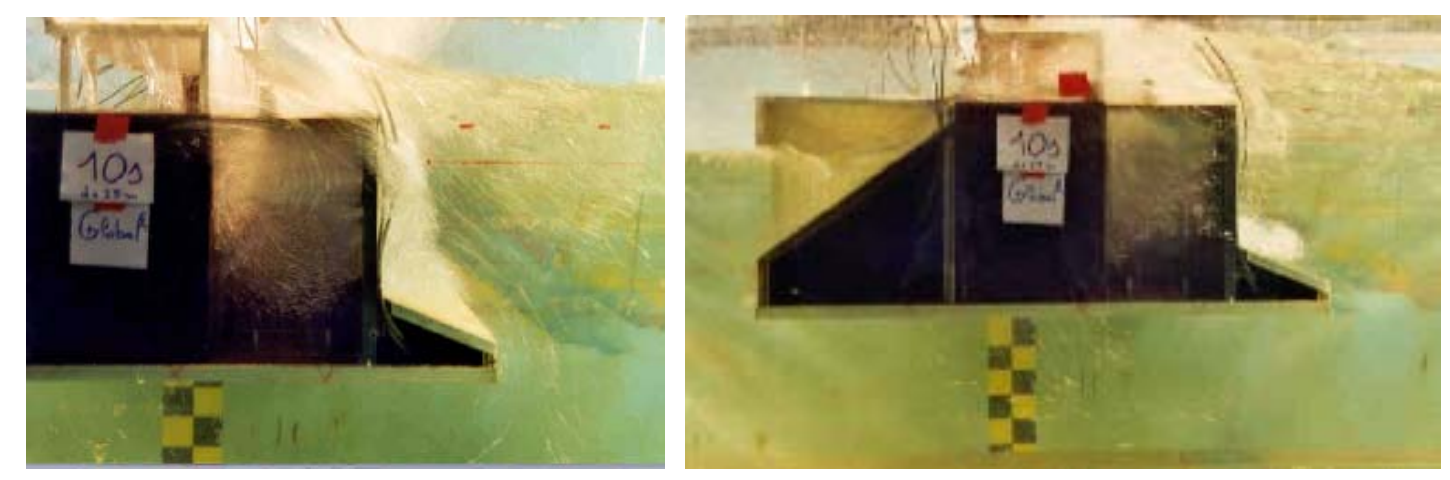

Figure 13 Vue des projections d'eau sur le mur du fond du Jarlan.

En effet, les capteurs du mur du fond du Jarlan sont des plaques non raccordée au mur du Jarlan et l'eau peut circuler de part et d'autre de la surface de mesure. La mesure ne concerne donc que l'effort dynamique. L'effort hydrostatique est calculé à partir de la sonde placée devant le mur et de la mesure de pression réalisée au droit du mur avec la formule suivante :

Fchambre $=\left[\frac{S_{11}}{2}+T e\right] \times P_{3} \times 9.81 \times 40^{2}$

Avec Te le tirant d'eau de la digue, S11 la sonde et P3 le capteur de pression. 


\subsection{Analyse de la composante " quasi-hydrostatique » et de la composante « impact " des efforts locaux principaux}

Le tableau 1 montre les valeurs principales maximales des efforts locaux horizontaux pour la configuration testée. Les parties « quasi-hydrostatiques » et « impacts » sont extraites de l'effort local. L'effort quasi-hydrostatique est mesuré juste après le pic d’impact.

Tableau 1 Comparaison efforts dynamique et effort quasi hydrostatique.

\begin{tabular}{|c|c|c|c|}
\hline & $\begin{array}{l}\text { Effort total à l'impact et \% de l'effort Global } \\
\qquad(\mathrm{KN} / \mathrm{ml})\end{array}$ & $\begin{array}{c}\text { Impact seul et } \\
\% \text { de l'effort local } \\
(\mathrm{KN} / \mathrm{ml})\end{array}$ & $\begin{array}{c}\text { Effort sans l'impact et } \\
\% \text { de l'effort local } \\
\text { (KN/ml) }\end{array}$ \\
\hline $\begin{array}{l}\text { Mur du fond } \\
\text { de la chambre }\end{array}$ & $\begin{array}{l}560 \\
75 \%\end{array}$ & $\begin{array}{c}210 \\
37.5 \%\end{array}$ & $\begin{array}{l}350 \\
62.5 \%\end{array}$ \\
\hline $\begin{array}{l}\text { Partie supérieure de } \\
\text { la paroi perforée }\end{array}$ & $\begin{array}{l}144 \\
19 \%\end{array}$ & $\begin{array}{c}48 \\
34 \%\end{array}$ & $\begin{array}{c}96 \\
66 \%\end{array}$ \\
\hline $\begin{array}{l}\text { Partie inférieure de } \\
\text { la paroi perforée }\end{array}$ & $\begin{array}{l}110 \\
15 \%\end{array}$ & $\begin{array}{c}49 \\
44 \%\end{array}$ & $\begin{array}{c}61 \\
56 \%\end{array}$ \\
\hline $\begin{array}{l}\text { Paroi perforée } \\
\text { Complète }\end{array}$ & $\begin{array}{c}254 \\
33 \%\end{array}$ & 97 & 157 \\
\hline
\end{tabular}

On constate que la partie de l'effort due au choc de la vague est de l'ordre de 38\% de l'effort mesuré sur le mur perforé et sur le mur du fond de la chambre. Le mur du fond de la chambre est la partie qui reprend le plus d'effort (75\% de Fx global) et l'étude de l'effort quasi-hydrostatique permet une bonne approche de l'ordre de grandeur de l'effort global (50\% de Fx global). Une formulation approchée de l'effort quasi-hydrostatique permet également d'avoir l'ordre de grandeur de l'effort global:

Fhydro $=\frac{H i}{2} \times(1+K r) \times\left(\left(\frac{1}{2} \times \frac{H i}{2} \times(1+K r)\right)+T e\right)$

On trouve pour nos conditions Fhydro $=450 \mathrm{~K}$ Newtons.

\subsection{Cumul des efforts horizontaux}

Une fois le calcul des efforts ponctuels réalisé et mis à l'échelle réelle, il est possible de les additionner et de comparer ce cumul avec le signal global traité.

On trouve un bon accord comme le montre la figure 14. La même technique de traitement a été réalisée sur les efforts verticaux et l'on trouve un bon accord également de superposition des efforts avec l'effort global. Les efforts globaux verticaux ne présentent pas de pic particulier hormis la pression mesurée à l’intérieur de la chambre perforée. 

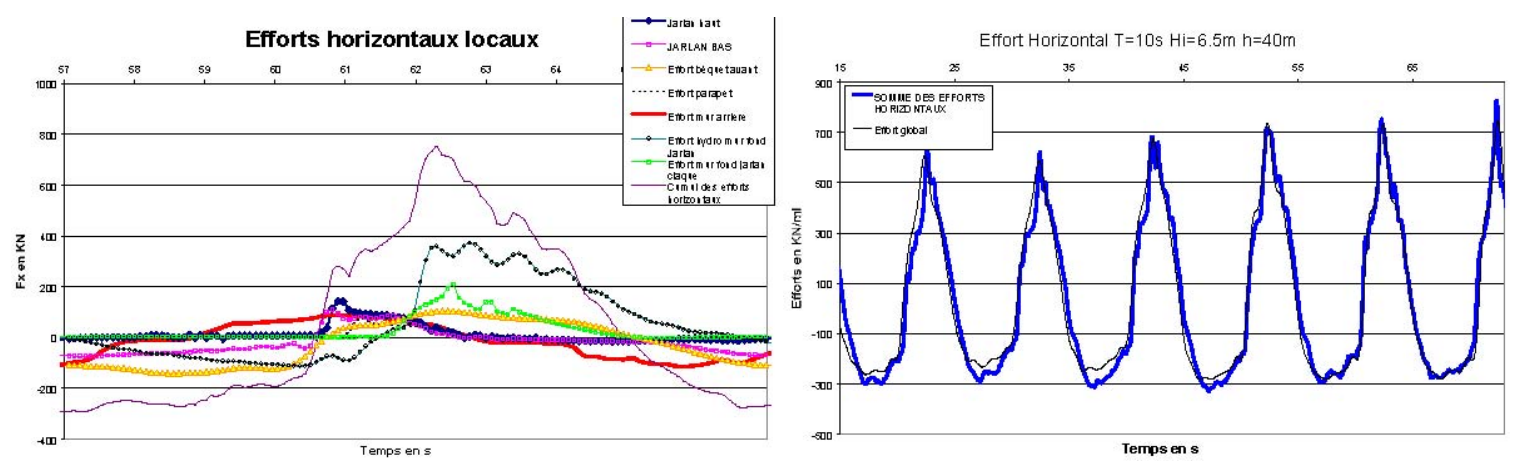

Figure 14 Cumul des efforts horizontaux locaux et comparaison avec l'effort horizontal global
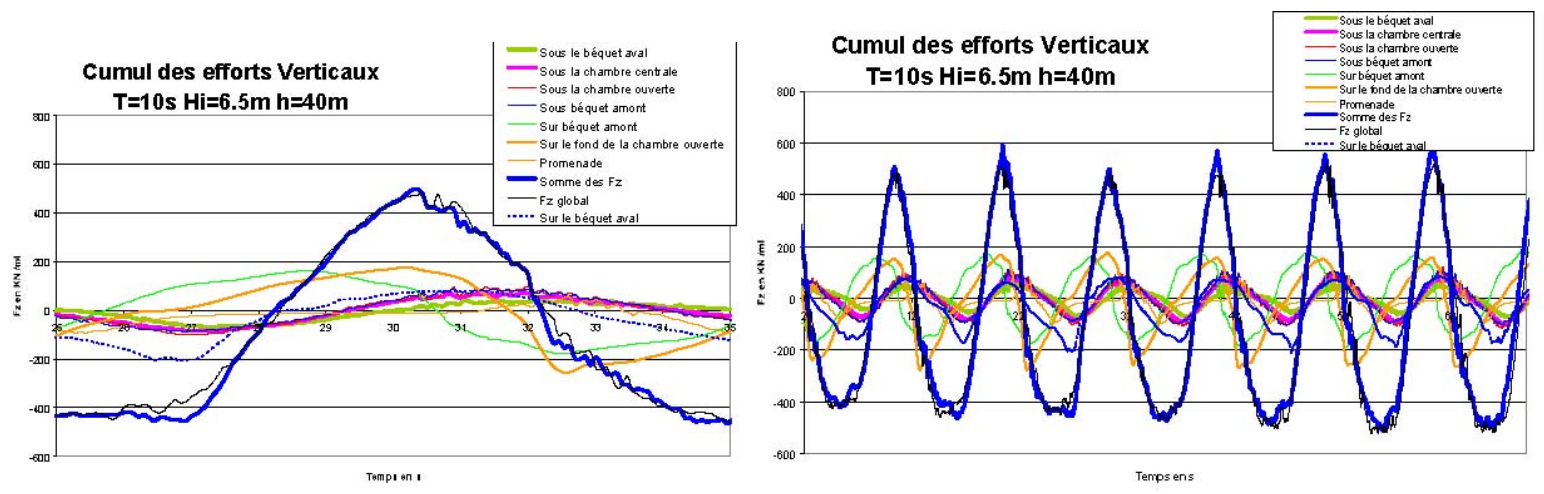

Figure 15 Efforts verticaux cumulés en réel

\section{Conclusion.}

On constate qu'à partir des essais réalisés au laboratoire Océanide, il nous a été possible de comprendre le phénomène dynamique induit par la houle sur la contre-jetée. Nous avons observé que les phénomènes liés aux inclusions d'air sont ponctuels et n'apparaissent pas au niveau de l'effort global. Localement, leur transposition à l'échelle réelle par la méthode de Lungreen montre qu'ils ne sont pas importants dans la réalité. Inversement nous avons observé que les oscillations de la maquette (due à la balance de mesure d'efforts globaux) influaient sur les efforts locaux.

Concernant la contribution des différents efforts locaux à la reconstitution de l'effort global, nous remarquons que la part liée aux différences de hauteur de surface libre est prépondérante par rapport à celle due aux impacts. Une estimation rapide de ces hauteurs peut donner un ordre de grandeur sur l'effort global qu'il faudra prendre en compte. 


\section{Références.}

1.Bouchet R., 1986, Techniques nouvelles de brise houle et de protection des sites côtiers. Le mur d'eau, dispositif nouveau de brise-houle en eau profonde, $p^{\circ} 60$ PIANC AIPCN Bulletin $n^{\circ} 52$.

2.Bélorgey M. \& Colmard C., 1998, Digue Partielle à profil optimisé : Système Bybop , 5em journées Génie Civil Génie Côtier Toulon.

3.Lesprit I. Fremy N. ,1999, Essais en canal à houle, Contre-jetée de Monaco, Rapport final.

4.Lundgren H., 1969, Wave Shock Force : An analysis of deformations and forces in the wave and in the foundation, Symposium on wave action, vol II Paper 4, Delft

5.Colmard C., 2000, Contre-jetée, analyse des essais hydrauliques, 1MCA 70 RA332 Rev0, Rapport

interne. 